\title{
Relation of Enteric $\alpha$-Synuclein to Gastrointestinal Dysfunction in Patients With Parkinson's Disease and in Neurologically Intact Subjects
}

\begin{abstract}
Hyo Jeong Lee, ${ }^{1}$ Kee Wook Jung, ${ }^{2}$ Sun Ju Chung, ${ }^{3}$ Seung-Mo Hong, ${ }^{4}$ Juyeon Kim, ${ }^{3}$ Jeong Hoon Lee, ${ }^{2}$ Sung Wook Hwang, ${ }^{2}$ Ho-Sung $\mathrm{Ryu}^{3}{ }^{3}$ Mi Jung Kim, ${ }^{1}$ Ho-Su Lee, ${ }^{5}$ Myeongsook Seo, ${ }^{2}$ Sang Hyoung Park, ${ }^{2}$ Dong-Hoon Yang, ${ }^{2}$ Byong Duk Ye, ${ }^{2}$ Jeong-Sik Byeon, ${ }^{2}$ Jaewon Choe, ${ }^{1,2}$ Hwoon-Yong Jung, ${ }^{2}$ Suk-Kyun Yang, ${ }^{2}$ and Seung-Jae Myung ${ }^{2,6 *}$

${ }^{1}$ Health Screening and Promotion Center, Asan Medical Center, University of Ulsan College of Medicine, Seoul, Korea; Departments of ${ }^{2}$ Gastroenterology, ${ }^{3}$ Neurology, and ${ }^{4}$ Pathology, Asan Medical Center, University of Ulsan College of Medicine, Seoul, Korea; ${ }^{5}$ Department of Biochemistry and Molecular Biology, University of Ulsan College of Medicine, Seoul, Korea; and ${ }^{6}$ Asan Institute for Life Sciences, Asan Medical Center, Seoul, Korea
\end{abstract}

\section{Background/Aims}

$\alpha$-Synucleinopathy in the brain is the neuropathological hallmark of Parkinson's disease (PD). However, the functional impact of $\alpha$-synucleinopathy in the enteric nervous system remains unknown. We aim to evaluate the association between gastrointestinal (GI) dysfunction and $\alpha$-synuclein ( $\alpha S Y N)$ pathology in the stomach and colon of PD patients and controls, as well as to investigate the association between the $\alpha$ SYN pathology in GI tract and future PD risk.

\section{Methods}

A total of 35 PD patients and 52 neurologically intact subjects were enrolled in this study. Endoscopic biopsies were performed, and then immunohistochemical staining for $\alpha \mathrm{SYN}$ was performed. All subjects completed the validated Rome III questionnaire for the assessment of GI symptoms. The association between GI symptoms and the $\alpha$ SYN pathology in GI mucosa was evaluated. Incident PD cases were assessed during a median follow-up of 46 months.

\section{Results}

The proportion of self-reported constipation and functional constipation through the Rome III questionnaire was significantly higher in PD patients than in controls $(P<0.001$ and $P=0.015)$. However, no significant association was found between the $\alpha$ SYN pathology in the stomach and colon mucosa and constipation, as well as other $\mathrm{Gl}$ symptoms including dyspepsia symptoms and abdominal discomfort or pain, regardless of the presence or absence of clinical PD $(P>0.05)$. No incident PD cases were diagnosed during study period.

\section{Conclusions}

Our present study suggests that the deposition of $\alpha \mathrm{SYN}$ in the mucosal enteric nervous system may not be reflected by functional impairment of the affected segment of the gut.

(J Neurogastroenterol Motil 2018;24:469-478)

Key Words

$\alpha$-Synuclein; Enteric nervous system; Gastrointestinal symptoms; Parkinson disease

Received: December 14, 2017 Revised: February 19, 2018 Accepted: April 10, 2018

(.) This is an Open Access article distributed under the terms of the Creative Commons Attribution Non-Commercial License (http://creativecommons. org/licenses/by-nc/4.0) which permits unrestricted non-commercial use, distribution, and reproduction in any medium, provided the original work is properly cited.

*Correspondence: Seung-Jae Myung, MD, PhD, AGAF

Department of Gastroenterology and Asan Institute for Life Sciences, Asan Medical Center, University of Ulsan College of Medicine, 88 Olympic-ro 43-gil, Songpa-gu, Seoul 05505, Korea

Tel: +82-2-3010-3917, Fax: +82-2-476-0824, E-mail: sjmyung@amc.seoul.kr 


\section{Introduction}

Gastrointestinal (GI) symptoms are the most prominent and disabling non-motor manifestations of Parkinson's disease (PD). ${ }^{1}$ Patients with PD patients develop various GI symptoms, including dry mouth, drooling, dysphagia, gastroparesis, and constipation. ${ }^{1,2}$ Although the clinical understanding of GI dysfunction in PD patients has increased substantially, the pathophysiological mechanism of GI involvement in PD is still unclear.

$\alpha$-Synuclein $(\alpha \mathrm{SYN})$ is the main component of Lewy bodies and Lewy neurites, which are the main neuropathological hallmarks of PD. ${ }^{3}$ The abnormal deposition of aggregated $\alpha \mathrm{SYN}$ initially occurs in the olfactory bulb and dorsal motor nucleus of the vagus nerve (DMV) in the medulla; thereafter, it spreads through less vulnerable nuclear gray and cortical areas in an ascending course. ${ }^{4}$ Researchers have also found $\alpha \mathrm{SYN}$ aggregation in the enteric nervous system (ENS), occurring at the earliest stage of PD, or even preceding the onset of $\mathrm{PD} .^{5-7}$ GI symptoms also occur from the earliest stage of $\mathrm{PD}$, and constipation is known to precede the motor symptoms of the disease. ${ }^{1,8}$ In addition, the extent of GI dysfunction corresponds with widespread ENS synucleinopathy, suggesting that the abnormal deposition of $\alpha \mathrm{SYN}$ in the ENS could be the main cause of GI dysfunction in PD. ${ }^{9}$ However, no studies have shown a causative link between this pathological abnormality and the corresponding GI deficit in $\mathrm{PD}{ }^{2}$

Several recently published studies have reported that $\alpha \mathrm{SYN}$ is also present in the colonic mucosa or submucosa of persons without neurodegenerative diseases. ${ }^{10-12}$ Our group also reported that there was no difference in the $\alpha \mathrm{SYN}$ immunoreactivity of gastric and colonic mucosal tissues obtained through routine endoscopy among PD patients and controls. ${ }^{13}$ However, the functional impact of $\alpha \mathrm{SYN}$ pathology in the GI tract of neurologically intact persons remains unknown. The role of ENS synucleinopathy in neurologically intact persons in predicting future PD risk has also not been evaluated.

Therefore, in the present study, we aim to evaluate the association between the $\alpha \mathrm{SYN}$ pathology in the human GI tract, and GI dysfunction in PD patients and neurologically intact persons, as well as to investigate the association between the $\alpha \mathrm{SYN}$ pathology in the GI tract and the subsequent risk of PD in neurologically intact subjects.

\section{Materials and Methods}

\section{Study Population}

Our group's preceding study was a prospective study comparing $\alpha \mathrm{SYN}$ immunoreactivity between PD and controls (neurologically intact subjects). ${ }^{13}$ In this study, the sample population comprised of 87 subjects who completed the validated Korean Rome III questionnaire (Rome III-K) among the participants of the preceding study. ${ }^{13,14}$ We enrolled PD patients and controls between August 2013 and February 2015. The PD patients were enrolled from the clinical practice of the Department of Neurology at Asan Medical Center, a tertiary university hospital in Seoul, South Korea, and the diagnosis of $\mathrm{PD}$ was made by experienced movement disorder specialists by using the clinical diagnostic criteria of the United Kingdom PD Society Brain Bank. ${ }^{15}$ Control subjects older than 45 years, without a history of neurological disorders, and who visited the gastroenterology outpatient clinic at the Asan Medical Center were prospectively enrolled in this research. We screened all of the controls for PD by using the "Nine-symptom screening tool," ${ }^{\prime 16}$ and the subjects were examined by neurologists (S.J.C., J.K., H.S.R., and M.J.K.) to exclude neurological disorders. The control subjects had no psychological diseases, no tremors, and no impairment in cognitive function or the activities of daily living. Subjects were excluded if they had chronic GI diseases, such as Crohn's disease or ulcerative colitis, or if they showed active GI diseases in their esophagogastroduodenoscopy (EGD) or colonoscopy, such as a gastric ulcer, gastritis, colitis, or cancer.

\section{Endoscopic Biopsy}

The enrolled subjects underwent an EGD, colonoscopy, or both, as recommended by the gastroenterologist in routine clinical practice. An endoscopy was performed by 3 gastroenterologists (H.J.L., K.W.J., and J.H.L.) according to the usual procedure followed by the Gastroenterology Department of Asan Medical Center. We performed gastric mucosal biopsies in the fundus and antrum by using standard biopsy forceps (FB-25K-1; standard fenestrated cups; Olympus, Tokyo, Japan). Colonic mucosal biopsies were also performed in the ascending colon, transverse colon, and sigmoid colon by using standard biopsy forceps (FB-24U-1; standard fenestrated cups with needle, Olympus). Two random biopsies were taken from each biopsy site, and the two biopsies obtained from the same site were considered as a single sample. After sampling, the gastric and colonic mucosal specimens were immediately 

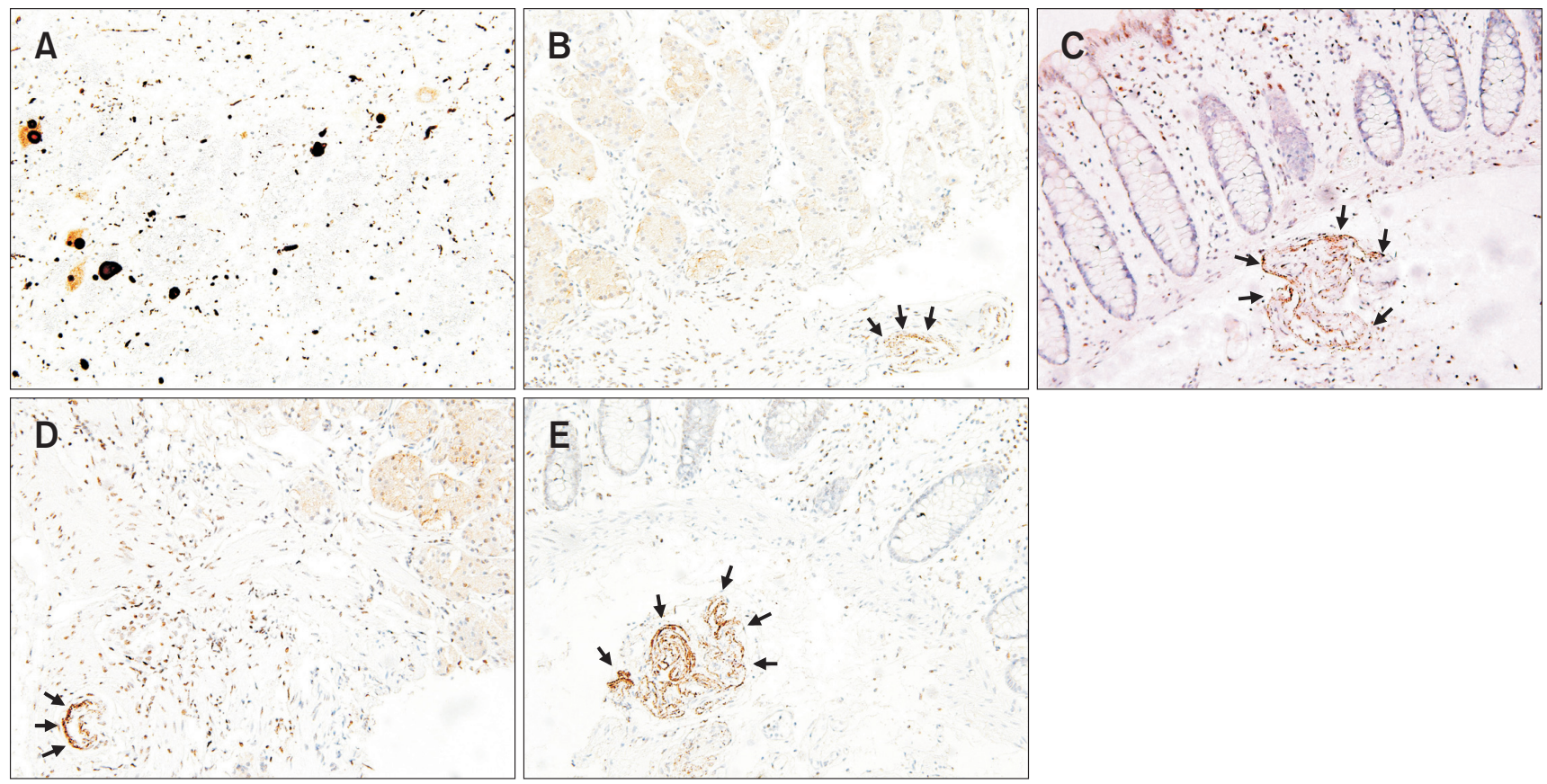

Figure. $\alpha$-Synuclein $(\alpha \mathrm{SYN})$ immunostaining. (A) Positive control of $\alpha \mathrm{SYN}$ immunoreactivity in this study; Lewy body-like inclusions and Lewy neurites with strong $\alpha \mathrm{SYN}$ immunoreactivity in brain tissue from autopsy of a patient with Parkinson's disease. (B-E) $\alpha \mathrm{SYN}$ immunostaining in gastric and colonic endoscopic biopsy tissues. (B) Gastric tissue from a 78-year-old man with an 11-year history of Parkinson's disease. (C) Colonic tissue from a 55-year-old woman with a 2-year history of Parkinson's disease (D) Gastric tissue from a 67-year-old woman as a control. (E) Colonic tissue from an 80-year-old man as a control. Note the positive $\alpha \mathrm{SYN}$ immunoreactivity reveals multiple dots-like or short-linear appearance (arrows), which is located close to the muscularis mucosa (all figures, original magnification, $\times 200$ ).

fixed in $10 \%$ formalin.

Our study protocol was approved by the institutional review board of Asan Medical Center (Protocol No. 2013-0175), and informed consent was obtained from all subjects.

\section{Immunohistochemical Staining and Tissue Assessment Procedures}

The primary antibodies for $\alpha \mathrm{SYN}$ (1:200; EP1536Y; ABCAM, Cambridge, UK) and S-100 protein (1:200; 18-0046; Zymed, South San Francisco, CA, USA), with BenchMark autostainers (Ventana Medical Systems, Tuscan, AZ, USA), were used according to the manufacturers' protocol. Then, $4-\mu \mathrm{m}$ tissue sections were deparaffinized, and hydrated in xylene and serially diluted ethanol, respectively. Endogenous peroxidase was blocked by incubation in $3 \% \mathrm{H}_{2} \mathrm{O}_{2}$ for 10 minutes, after which heat-induced antigen retrieval was performed for 32 minutes. After primary antibody incubation for 32 minutes at room temperature, detection was carried out with an I-View detection kit (BenchMark XT, Ventana Medical Systems).

The specimens were determined to be adequate for $\alpha \mathrm{SYN}$ im- munostaining when sufficient numbers of sections were available for analysis, and the density of S-100-positive nerve cells was at least low to moderate, with the presence of the muscularis mucosa. A section with at least one definite $\alpha \mathrm{SYN}$ immunoreactive fiber was considered to be positive when compared with the positive control of $\alpha \mathrm{SYN}$ immunoreactivity (ie, Lewy body-like inclusions or Lewy neurites from the brain tissues of PD patients; Fig. A). The neuropathological findings were assessed by one neuropathologist (S.M.H.) and one experienced neurologist (J.K.) who were blinded to the clinical information of subjects.

\section{Assessment of Gastrointestinal Symptoms}

All subjects completed the validated Korean Rome III questionnaire to assess their GI symptoms (Rome III-K). ${ }^{14}$ The questionnaires evaluated the frequency and duration of the GI symptoms, including epigastric burning, early satiation, postprandial fullness, epigastric pain, acid reflux, nausea, vomiting, abdominal pain or discomfort, constipation, bloating, and fecal incontinence, during the previous 3 months. Each self-reported GI symptom was defined as "presence" if it met the following criteria set by Rome 
$\mathrm{III}^{17}$ : epigastric burning, epigastric pain, and vomiting one or more times per week; early satiation, postprandial fullness, and nausea from 2 to 3 or more times per week; and abdominal pain or discomfort and bloating for $\geq 3$ days/month. The presence of acid reflux was confirmed when the symptom occurred more than once per week, and the presence of fecal incontinence was confirmed when the symptom occurred more than once per month. The presence of self-reported constipation was confirmed when the subjects reported defecatory difficulty. Functional GI disorders (functional dyspepsia, irritable bowel syndrome, and functional constipation) were diagnosed as defined by the Rome III criteria. In the PD patients, the presence of drooling and dysphagia were also assessed by using the non-motor symptom assessment scale for PD. ${ }^{18}$

We evaluated the association between the presence of GI symptoms and the $\alpha \mathrm{SYN}$ pathology of the GI tract in PD patients and neurologically intact subjects. We also assessed the relation between upper GI symptoms and $\alpha \mathrm{SYN}$ in the gastric samples, as well as the relation between lower GI symptoms and $\alpha \mathrm{SYN}$ in the colon samples. This assessment sought to evaluate the organ-specific association between the $\alpha \mathrm{SYN}$ pathology and GI symptoms. Upper GI symptoms included drooling, dysphagia, epigastric burning, early satiation, postprandial fullness, epigastric pain, acid reflux, nausea, and vomiting. Lower GI symptoms included abdominal pain or discomfort, constipation, bloating, and fecal incontinence.

\section{Ascertainment of Parkinson's Disease}

The outcome of interest was defined as having a disease code for expanding benefit coverage for PD patients' (V124) registration with the Korean National Health Insurance Service.

\section{Statistical Methods}

The differences in the continuous variables between the groups were evaluated by using the Mann-Whitney $U$ test, and the differences in the categorical variables were evaluated with the $\chi^{2}$ test or Fisher's exact test. Data were analyzed with SPSS 20.0 (IBM Corp, Armonk, NY, USA), and $P$-values $<0.05$ were considered to be statistically significant.

\section{Results}

\section{Baseline Characteristics of the Study Population}

A total of 35 PD patients and 52 controls were enrolled in this study, and the baseline characteristics and GI symptoms of the subjects are shown in Table 1 . There were no significant differences between the two groups in terms of age, sex, and body mass index.

Among the $35 \mathrm{PD}$ patients, the median disease duration was 7 years (range, 2-21 years), the mean levodopa equivalent dose was $816 \mathrm{mg} /$ day, the mean Hoehn and Yahr stage was 2.4, and the mean total unified PD rating scale score was 40.9. Three patients (8.6\%) underwent EGD only, 16 (45.7\%) underwent colonoscopy only, and 16 (45.7\%) underwent both procedures.

Among the 52 controls, 21 subjects (40.4\%) were given screening endoscopies without GI symptoms. The main reasons for performing endoscopy in the remaining controls $(n=31,59.6 \%)$ were as follows: constipation ( $\mathrm{n}=18$ ), abdominal pain or discomfort $(n=6)$, acid reflux $(n=2)$, epigastric pain $(n=1)$, postprandial discomfort $(\mathrm{n}=1)$, bloating $(\mathrm{n}=1)$, and diarrhea $(\mathrm{n}=2)$. Seven subjects $(13.5 \%)$ underwent EGD only, 24 (46.2\%) underwent colonoscopy only, and 21 (40.4\%) underwent both procedures.

The rates of self-reported constipation and functional constipation through the Rome III questionnaire were significantly higher in $\mathrm{PD}$ patients than in controls $(P<0.001$ and $P=0.015)$. The other self-reported GI symptoms and functional GI disorders did not differ between the two groups.

\section{$\alpha$-Synuclein in the Gastric or Colonic Mucosa Is Not Associated With Gastrointestinal Symptoms}

Of the 87 subjects, 79 (90.8\%) had one or more adequate quality samples, and were assessable for neuropathological study; $\alpha \mathrm{SYN}$ was documented in 32 subjects (40.5\%). $\alpha \mathrm{SYN}$ immunostaining in the gastric and colonic endoscopic biopsy tissue were illustrated in Figure B-E. There was no difference in the $\alpha \mathrm{SYN}$ immunoreactivity between the controls and $\mathrm{PD}$ patients $(43.5 \%$ vs $36.4 \%, P=0.643$; Supplementary Table 1).

The GI symptoms of the subjects according to the $\alpha \mathrm{SYN}$ immunostaining in the mucosal biopsies, regardless of the presence or absence of an underlying neurological disorder, are indicated in Table 2. No statistically significant differences were found in the demographic variables, self-reported GI symptoms, or functional GI disorders in Rome III between the negative $\alpha \mathrm{SYN}$ group $(\mathrm{n}=$ 47 ) and the positive $\alpha \mathrm{SYN}$ group $(\mathrm{n}=32)$. In addition, $\alpha \mathrm{SYN}$ positivity in gastric mucosal biopsy was not associated with any upper or lower GI symptoms (Supplementary Table 2), and the results according to the $\alpha \mathrm{SYN}$ positivity in the colonic mucosal biopsy were the same (Supplementary Table 3). Moreover, the detailed constipation symptoms did not differ between the two groups (Supplementary Table 4).

The GI symptoms of PD patients according to $\alpha \mathrm{SYN}$ immunostaining in the mucosal biopsy are indicated in Table 3. Among 
Table 1. Baseline Characteristics and Gastrointestinal Symptoms of the Study Subjects

\begin{tabular}{|c|c|c|c|}
\hline Variables & Control $(\mathrm{n}=52)$ & $\mathrm{PD}(\mathrm{n}=35)$ & $P$-value \\
\hline Age (median [range], yr) & $63(50-80)$ & $67(50-79)$ & 0.098 \\
\hline Sex (male, $n)$ & $26(50.0 \%)$ & $15(42.9 \%)$ & 0.513 \\
\hline Body mass index (median [range], $\mathrm{kg} / \mathrm{m}^{2}$ ) & $24(18-34)$ & $22(17-35)$ & 0.090 \\
\hline \multicolumn{4}{|l|}{ Characteristics of PD } \\
\hline Duration of disease (median [range], yr) & - & $7(2-21)$ & \\
\hline Levodopa equivalent dose (mean $\pm \mathrm{SD}, \mathrm{mg} /$ day) & - & $816 \pm 339$ & \\
\hline Hoehn and Yahr stage (mean $\pm \mathrm{SD}$ ) & - & $2.4 \pm 0.6$ & \\
\hline \multicolumn{4}{|l|}{ UPDRS of PD $($ mean $\pm \mathrm{SD})$} \\
\hline Part I & - & $2.9 \pm 2.3$ & \\
\hline Part II & - & $11.8 \pm 5.6$ & \\
\hline Part III & - & $23.2 \pm 9.5$ & \\
\hline Part IV & - & $3.1 \pm 2.9$ & \\
\hline Total & - & $40.9 \pm 16.8$ & \\
\hline \multicolumn{4}{|l|}{ Self-reported GI symptoms (n) } \\
\hline Epigastric burning & $5(9.6 \%)$ & $2(5.7 \%)$ & 0.697 \\
\hline Early satiation & $9(17.3 \%)$ & $3(8.6 \%)$ & 0.347 \\
\hline Postprandial fullness & $10(19.2 \%)$ & $2(5.7 \%)$ & 0.112 \\
\hline Epigastric pain & $6(11.5 \%)$ & $3(8.6 \%)$ & 0.735 \\
\hline Acid reflux & $6(11.5 \%)$ & $1(2.9 \%)$ & 0.234 \\
\hline Nausea & $1(1.9 \%)$ & $2(5.7 \%)$ & 0.562 \\
\hline Vomiting & $0(0.0 \%)$ & $1(2.9 \%)$ & 0.402 \\
\hline Abdominal pain/discomfort & $13(25.0 \%)$ & $3(8.6 \%)$ & 0.088 \\
\hline Constipation & $24(46.2 \%)$ & $32(91.4 \%)$ & $<0.001$ \\
\hline Bloating & $14(26.9 \%)$ & $6(17.1 \%)$ & 0.288 \\
\hline Fecal incontinence & $3(5.8 \%)$ & $2(5.7 \%)$ & 1.000 \\
\hline \multicolumn{4}{|l|}{ Functional GI disorder (Rome III) (n) } \\
\hline Functional dyspepsia & $12(23.1 \%)$ & $5(14.3 \%)$ & 0.412 \\
\hline Irritable bowel syndrome & $6(11.5 \%)$ & $1(2.9 \%)$ & 0.234 \\
\hline Functional constipation & $25(48.1 \%)$ & $26(74.3 \%)$ & 0.015 \\
\hline
\end{tabular}

PD, Parkinson's disease; SD, standard deviation; UPDRS, unified Parkinson's disease rating scale; GI, gastrointestinal.

the PD patients, 33 (94.3\%) were assessable for neuropathological study, and $\alpha \mathrm{SYN}$ was documented in 12 (36.4\%) patients. More $\mathrm{PD}$ patients with negative $\alpha \mathrm{SYN}$ had self-reported constipation than those with positive $\alpha \mathrm{SYN}(100 \%$ vs $75 \%, P=0.040)$, whereas functional constipation according to the Rome III criteria did not differ between the two groups. The other self-reported GI symptoms and functional GI disorders did not differ between the two groups.

The GI symptoms of the neurologically intact subjects, according to the $\alpha \mathrm{SYN}$ immunostaining in the mucosal biopsy, are indicated in Table 4. No statistically significant differences were found in the demographic variables, self-reported GI symptoms, or functional GI disorders in Rome III between the negative $\alpha \mathrm{SYN}$ group $(\mathrm{n}=26)$ and the positive $\alpha \mathrm{SYN}$ group $(\mathrm{n}=20)$.

\section{The Association Between $\alpha$-Synuclein Pathology in the Gastrointestinal Tract and Future Parkinson's Disease Risk During Short-term Follow-up Was Not Assessed}

No incident PD cases were diagnosed during a median follow up of 46 months (range 31-52) among 52 baseline neurologically intact subjects, irrespective of their GI $\alpha \mathrm{SYN}$ pathology. The association between the $\alpha \mathrm{SYN}$ pathology in the GI tract and future PD risk could not be evaluated.

\section{Discussion}

Our present study described the association between the $\alpha \mathrm{SYN}$ pathology in the human GI mucosa, and GI dysfunction in $\mathrm{PD}$ patients and neurologically intact subjects. The $\alpha \mathrm{SYN}$ pathol- 
Table 2. Gastrointestinal Symptoms of Subjects With Positive and Negative $\alpha$-Synuclein Immunostaining in the Stomach and Colon Mucosal Biopsies

\begin{tabular}{lccc}
\hline \multicolumn{1}{c}{ Variables } & Negative $(\mathrm{n}=47)$ & Positive $^{\mathrm{a}}(\mathrm{n}=32)$ & $P$-value \\
\hline Age (median [range], yr) & $65(50-77)$ & $66(50-80)$ & 0.301 \\
Sex (male, $\mathrm{n}$ ) & $25(53.2 \%)$ & $17(53.1 \%)$ & 0.995 \\
Body mass index (median [range], $\left.\mathrm{kg} / \mathrm{m}^{\mathrm{b}}\right)$ & $23(17-35)$ & $24(18-29)$ & 0.830 \\
Self-reported GI symptoms (n) & & & 0.152 \\
Epigastric burning & $1(2.1 \%)$ & $4(12.5 \%)$ & 0.515 \\
Early satiation & $5(10.6 \%)$ & $5(15.6 \%)$ & 1.000 \\
Postprandial fullness & $7(14.9 \%)$ & $4(12.5 \%)$ & 0.216 \\
Epigastric pain & $2(4.3 \%)$ & $4(12.5 \%)$ & 0.682 \\
Acid reflux & $3(6.4 \%)$ & $3(9.4 \%)$ & 0.563 \\
Nausea & $1(2.1 \%)$ & $2(6.3 \%)$ & 0.405 \\
Vomiting & $0(0.0 \%)$ & $1(3.1 \%)$ & 0.358 \\
Abdominal pain/discomfort & $6(12.8 \%)$ & $17(53.1 \%)$ & 0.058 \\
Constipation & $35(74.5 \%)$ & $6(18.8 \%)$ & 0.589 \\
Bloating & $12(25.5 \%)$ & $3(9.4 \%)$ & 0.390 \\
Fecal incontinence & $2(4.3 \%)$ & & 1.000 \\
Functional GI disorder (Rome III) (n) & & $5(15.6 \%)$ & 1.000 \\
Functional dyspepsia & $3(17.0 \%)$ & $2(6.3 \%)$ & 0.819 \\
Irritable bowel syndrome & $28(59.6 \%)$ & $20(62.5 \%)$ & \\
Functional constipation & & & \\
\hline
\end{tabular}

${ }^{\mathrm{a}}$ Positive: $\alpha$-synuclein stain $(+) \geq 1$ site.

${ }^{b}$ Eight subjects without adequate samples were excluded from this analysis. GI, gastrointestinal.

ogy in the stomach and colon mucosa appeared not to be associated with GI dysfunction. Therefore, our findings suggested that the deposition of $\alpha \mathrm{SYN}$ in the stomach and colon mucosal nerve fiber may not be reflected by the functional impairment of the affected segment of the gut, regardless of the presence or absence of clinical PD.

GI dysfunction is the most prominent and disabling nonmotor manifestation of PD, and it is significantly more prevalent in $\mathrm{PD}$ patients than in controls. ${ }^{1}$ However, the current treatment strategy for GI dysfunction in PD patients has focused on symptomatic treatment. ${ }^{2,19}$ An understanding of the pathophysiological mechanism of GI motility dysfunction in PD would help in the development of target treatments for GI symptoms. However, the pathophysiological mechanism is still poorly understood, and even the clinical-pathological correlations between the GI symptoms and PD pathology have not been well evaluated.

The normal function of the GI tract is controlled by intrinsic and extrinsic innervation; intrinsic innervation relies on the ENS, and extrinsic innervation depends on the preganglionic parasympathetic and sympathetic outputs. ${ }^{20,21}$ The parasympathetic output originates in the DMV of the medulla and in the sacral parasympa- thetic nucleus of the spinal cord. ${ }^{20,21}$ The ENS is especially thought to play a major role in GI dysfunction in PD. ${ }^{9}$ The ENS has essential roles in controlling bowel movement and secretion, largely independently of influences from the central nervous system, and the widespread distribution of $\alpha \mathrm{SYN}$ pathology in the ENS seems to be associated with various symptoms throughout the entire GI tract. $^{9,21}$ One clinical study reported a positive correlation between the amount of Lewy neurites in the submucosal plexus of the ENS and the severity of constipation in PD patients, suggesting a pathogenic role for ENS synucleinopathy in GI dysfunction in PD. ${ }^{22}$ However, no neuronal loss in the myenteric plexus in PD was reported, and the rostrocaudal distribution of the $\alpha \mathrm{SYN}$ burden in the ENS did not coincide with the characteristics of GI dysfunction in $\mathrm{PD}$, in which constipation is the most frequently occurring symptom, developing in the early phase. ${ }^{1,23,24}$ Therefore, further evaluation is required concerning the clinical-pathological correlations between ENS synucleinopathy and GI dysfunction in PD, and the possible mechanisms.

Furthermore, recent studies have reported that $\alpha \mathrm{SYN}$ was detected in the ENS of neurologically intact subjects; that is, it may not be a specific finding of PD patients. ${ }^{10-12,25}$ Our group also re- 
Table 3. Gastrointestinal Symptoms of Patients With Parkinson's Disease With Positive and Negative $\alpha$-Synuclein Immunostaining in the Stomach and Colon Mucosal Biopsies

\begin{tabular}{|c|c|c|c|}
\hline Variables & Negative $(\mathrm{n}=21)$ & $\operatorname{Positive~}^{\mathrm{a}}(\mathrm{n}=12)$ & $P$-value ${ }^{\mathrm{b}}$ \\
\hline Age (median [range], yr) & $67(51-77)$ & $66(50-79)$ & 0.567 \\
\hline Sex (male, $n)$ & $12(57.1 \%)$ & $7(58.3 \%)$ & 1.000 \\
\hline Body mass index (median [range], $\mathrm{kg} / \mathrm{m}^{2}$ ) & $22(17-35)$ & $22(18-26)$ & 0.645 \\
\hline \multicolumn{4}{|l|}{ Self-reported GI symptoms (n) } \\
\hline Drooling & $7(35.0 \%)$ & $3(25.0 \%)$ & 0.703 \\
\hline Dysphagia & $3(15.0 \%)$ & $1(8.3 \%)$ & 1.000 \\
\hline Epigastric burning & $0(0.0 \%)$ & $2(16.7 \%)$ & 0.125 \\
\hline Early satiation & $2(9.5 \%)$ & $1(8.3 \%)$ & 1.000 \\
\hline Postprandial fullness & $1(4.8 \%)$ & $1(8.3 \%)$ & 1.000 \\
\hline Epigastric pain & $0(0.0 \%)$ & $2(16.7 \%)$ & 0.125 \\
\hline Acid reflux & $0(0.0 \%)$ & $1(8.3 \%)$ & 0.364 \\
\hline Nausea & $0(0.0 \%)$ & $2(16.7 \%)$ & 0.125 \\
\hline Vomiting & $0(0.0 \%)$ & $1(8.3 \%)$ & 0.364 \\
\hline Abdominal pain/discomfort & $2(9.5 \%)$ & $1(8.3 \%)$ & 1.000 \\
\hline Constipation & $21(100.0 \%)$ & $9(75.0 \%)$ & 0.040 \\
\hline Bloating & $3(14.3 \%)$ & $2(16.7 \%)$ & 1.000 \\
\hline Fecal incontinence & $1(4.8 \%)$ & $1(8.3 \%)$ & 1.000 \\
\hline \multicolumn{4}{|l|}{ Functional GI disorder (Rome III) (n) } \\
\hline Functional dyspepsia & $2(9.5 \%)$ & $2(16.7 \%)$ & 0.610 \\
\hline Irritable bowel syndrome & $1(4.8 \%)$ & $0(0.0 \%)$ & 1.000 \\
\hline Functional constipation & $15(71.4 \%)$ & $9(75.0 \%)$ & 1.000 \\
\hline
\end{tabular}

${ }^{\mathrm{a}}$ Positive: $\alpha$-synuclein stain $(+) \geq 1$ site.

'Two Parkinson's disease patients without adequate samples were excluded from this analysis.

GI, gastrointestinal.

ported that the $\alpha \mathrm{SYN}$ positivity in the gastric and colonic mucosal biopsied samples was comparable between $38 \mathrm{PD}$ patients and 53 controls $(33.3 \%$ for the stomach and $18.5 \%$ for the colon in the controls). ${ }^{13}$ These studies have raised concerns about whether the $\alpha \mathrm{SYN}$ neuropathology is the main pathophysiology of GI dysfunction in PD patients. Moreover, it is not clear what is the role of $\alpha \mathrm{SYN}$ in the GI tract of neurologically intact subjects.

To our knowledge, this study is the first to describe the association between the $\alpha \mathrm{SYN}$ pathology in the GI tract (upper and lower) of living humans, and comprehensive GI dysfunction in both PD and neurologically intact subjects. In this study, both controls (with or without GI symptoms) and PD patients were enrolled, and the validated and structured Korean Rome III questionnaire (Rome III-K) was used to assess the GI symptoms systematically. ${ }^{14}$ Unfortunately, we could not find any significant association between the $\alpha \mathrm{SYN}$ pathology in the GI mucosa and GI dysfunction. One unexpected finding was that PD patients without $\alpha \mathrm{SYN}$ had higher rates of self-reported constipation than those with $\alpha \mathrm{SYN}$, which might be an incidental finding due to the low sample size of PD patients.
We postulate several reasons for the negative results of this study. First, the accumulation of $\alpha \mathrm{SYN}$ in the ENS may not be the primary cause of the GI dysfunction in PD. The $\alpha \mathrm{SYN}$ neuropathology affected not only the ENS but also the DMV, sacral parasympathetic neurons, and sympathetic preganglionic and ganglion neurons; therefore, the contribution of these factors to GI dysfunction in PD remains to be defined. ${ }^{1,26,27}$ In particular, the DMV is almost affected in PD, and neuronal loss in this nucleus has also been reported. ${ }^{4,28}$ The vagal innervation from the DMV is more heavily distributed in the esophagus and stomach than in the distal GI tract; therefore, the dysmotility of the esophagus and stomach is likely to be associated with the involvement of the DMV., ${ }^{4,929}$ Further studies to evaluate the contribution of other affected nervous system components to GI dysfunction are required. In addition, aging, mobility, and drugs used to treat the motor symptoms of $\mathrm{PD}$ are also considered to be factors related to GI dysfunction in PD. ${ }^{9,30}$ Second, pathological $\alpha \mathrm{SYN}$ distinct from physiological $\alpha \mathrm{SYN}$ might exist, and the immunohistochemical method used in our study may fail to distinguish between the 2 types. Böttner et $\mathrm{al}^{10}$ reported the presence of native $\alpha \mathrm{SYN}$ in surgical specimens of the 
Table 4. Gastrointestinal Symptoms of Neurologically Intact Subjects With Positive and Negative $\alpha$-Synuclein Immunostaining in the Stomach and Colon Mucosal Biopsies

\begin{tabular}{|c|c|c|c|}
\hline Variables & Negative $(n=26)$ & $\operatorname{Positive}^{\mathrm{a}}(\mathrm{n}=20)$ & $P$-value ${ }^{\mathrm{b}}$ \\
\hline Age (median [range], yr) & $62(50-76)$ & $66(51-80)$ & 0.276 \\
\hline Sex (male, $\mathrm{n})$ & $13(50.0 \%)$ & $10(50.0 \%)$ & 1.000 \\
\hline Body mass index (median [range], $\mathrm{kg} / \mathrm{m}^{2}$ ) & $23(18-34)$ & $25(20-29)$ & 0.485 \\
\hline \multicolumn{4}{|l|}{ Self-reported GI symptoms (n) } \\
\hline Epigastric burning & $1(3.8 \%)$ & $2(10.0 \%)$ & 0.572 \\
\hline Early satiation & $3(11.5 \%)$ & $4(20.0 \%)$ & 0.682 \\
\hline Postprandial fullness & $6(23.1 \%)$ & $3(15.0 \%)$ & 0.711 \\
\hline Epigastric pain & $2(7.7 \%)$ & $2(10.0 \%)$ & 1.000 \\
\hline Acid reflux & $3(11.5 \%)$ & $2(10.0 \%)$ & 1.000 \\
\hline Nausea & $1(3.8 \%)$ & $0(0.0 \%)$ & 1.000 \\
\hline Vomiting & $0(0.0 \%)$ & $0(0.0 \%)$ & $\mathrm{N} / \mathrm{A}$ \\
\hline Abdominal pain/discomfort & $4(15.4 \%)$ & $6(30.0 \%)$ & 0.292 \\
\hline Constipation & $14(53.8 \%)$ & $8(40.0 \%)$ & \\
\hline Bloating & $9(34.6 \%)$ & $4(20.0 \%)$ & 0.336 \\
\hline Fecal incontinence & $1(3.8 \%)$ & $2(10.0 \%)$ & 0.572 \\
\hline \multicolumn{4}{|l|}{ Functional GI disorder (Rome III) (n) } \\
\hline Functional dyspepsia & $6(23.1 \%)$ & $3(15.0 \%)$ & 0.711 \\
\hline Irritable bowel syndrome & $2(7.7 \%)$ & $2(10.0 \%)$ & 1.000 \\
\hline Functional constipation & $13(50.0 \%)$ & $11(55.0 \%)$ & 0.774 \\
\hline
\end{tabular}

${ }^{\mathrm{a}}$ Positive: $\alpha$-synuclein stain $(+) \geq 1$ site.

${ }^{\mathrm{b}} \mathrm{Six}$ neurologically intact subjects without adequate samples were excluded from this analysis.

GI, gastrointestinal.

colon in 13 subjects without neurodegenerative diseases, and they suggested that $\alpha \mathrm{SYN}$ in the ENS appears to be a normal finding and not an indicator of neurodegenerative processes. One recent interesting study demonstrated that distinct $\alpha \mathrm{SYN}$ strains display differential seeding capacities, inducing strain-specific pathologies and neurotoxic phenotypes. ${ }^{31}$ The authors reported that fibrils seem to be the major toxic strain, resulting in progressive motor impairment and cell death. Therefore, further studies are warranted to elaborate on the pathological $\alpha \mathrm{SYN}$ characteristics and their functional impact on GI dysfunction. Finally, it is possible that GI dysmotility might be present even in the absence of symptoms.

Another aspect of identifying the role of $\alpha \mathrm{SYN}$ pathology in the GI tract of neurologically intact patients was the possibility that GI $\alpha$ SYN pathology could predict PD risk prior to any motor signs of PD. Our study sought to prospectively investigate the association between the $\alpha \mathrm{SYN}$ pathology in the GI tract and the subsequent risk of PD in neurologically intact subjects. However, because no incident PD cases were observed in our study period, the assessment could not be done. Although previous studies have reported that $\alpha \mathrm{SYN}$ positivity in the GI tissues were obtained several years (2-20 years) prior to the onset of motor symptoms in $\mathrm{PD}$ patients, there have been neither large population-based nor prospective cohort studies conducted. ${ }^{6,32,33}$ Our results were difficult to conclude because of the short follow-up period; therefore, further follow up with our cohort is required.

Our study has the following limitations. First, we did not evaluate the deep layer of the GI tract, including the submucosal and myenteric plexus of the ENS, because we obtained the GI mucosal layer by using endoscopic biopsy forceps. This sampling method was intended to obtain enteric tissues in the living human efficiently, and its usefulness has been reported in the detection of enteric $\alpha \mathrm{SYN}$ in PD patients. ${ }^{34-36}$ The low quality of the samples obtained with endoscopic biopsy is also one of the limitations of this study, and this may have affected the results of the neuropathological analysis. Future studies to evaluate the $\alpha \mathrm{SYN}$ pathology in the submucosal and myenteric plexus in living humans, by using novel neuropathological methods, are needed. Second, the number of subjects with certain GI symptoms was low, and the severity of symptoms was not assessed.

In conclusion, our present study suggests that the deposition of $\alpha \mathrm{SYN}$ in the mucosal ENS may not be reflected by functional impairment of the affected segment of the gut. These data provide a background for further analyses of the $\alpha \mathrm{SYN}$ neuropathology and GI dysfunction. Further investigations are warranted to elucidate 
the pathological $\alpha \mathrm{SYN}$ strains in the ENS that are distinguishable from the physiological $\alpha \mathrm{SYN}$ strains, as well as their clinical implications, and the exact mechanism of GI motility dysfunction.

\section{Supplementary Material}

Note: To access the supplementary table mentioned in this article, visit the online version of Journal of Neurogastroenterology and Motility at http://www.jnmjournal.org/, and at https://doi. org/10.5056/jnm17141.

Financial support: This study was supported by a grant of the Korea Healthcare Technology R \& D Project, Ministry of Health and Welfare, Republic of Korea (No. HI14C2206 and No. HI15C3078), and the Ministry of Trade, Industry \& Energy (MOTIE, Korea) under Industrial Technology Innovation Program (No. 10063408, 'Development of channel-inserted convergence smart endoscopic system based on multispectral fluorescence imaging for precise diagnosis of digestive diseases').

\section{Conflicts of interest: None.}

Author contributions: Hyo Jeong Lee performed the procedures, analyzed the data, and wrote the manuscript; Kee Wook Jung and Jeong Hoon Lee performed the procedures and collected the data; Seung-Mo Hong performed the pathological analysis; Sun Ju Chung designed the study; Juyeon Kim collected the data, performed neurologic examinations, and performed the pathological analysis; Ho-Sung Ryu, Mi Jung Kim collected data and performed neurologic examinations; Sung Wook Hwang, Ho-Su Lee, Myeongsook Seo, Sang Hyoung Park, Dong-Hoon Yang, Byong Duk Ye, Jeong-Sik Byeon, Jaewon Choe, Hwoon-Yong Jung, and Suk-Kyun Yang revised the manuscript; and Seung-Jae Myung designed the study and revised the manuscript. All authors have approved the final draft submitted.

\section{References}

1. Cersosimo MG, Raina GB, Pecci C, et al. Gastrointestinal manifestations in parkinson's disease: prevalence and occurrence before motor symptoms. J Neurol 2013;260:1332-1338.

2. Fasano A, Visanji NP, Liu LW, Lang AE, Pfeiffer RF. Gastrointestinal dysfunction in parkinson's disease. Lancet Neurol 2015;14:625-639.

3. Spillantini MG, Schmidt ML, Lee VM, Trojanowski JQ, Jakes R, Goedert M. Alpha-synuclein in lewy bodies. Nature 1997;388:839-840.

4. Braak H, Del Tredici K, Rüb U, de Vos RA, Jansen Steur EN, Braak E.
Staging of brain pathology related to sporadic parkinson's disease. Neurobiol Aging 2003;24:197-211.

5. Braak H, de Vos RA, Bohl J, Del Tredici K. Gastric alpha-synuclein immunoreactive inclusions in meissner's and auerbach's plexuses in cases staged for parkinson's disease-related brain pathology. Neurosci Lett 2006;396:67-72.

6. Shannon KM, Keshavarzian A, Dodiya HB, Jakate S, Kordower JH. Is alpha-synuclein in the colon a biomarker for premotor parkinson's disease? Evidence from 3 cases. Mov Disord 2012;27:716-719.

7. Shannon KM, Keshavarzian A, Mutlu E, et al. Alpha-synuclein in colonic submucosa in early untreated parkinson's disease. Mov Disord 2012;27:709-715.

8. Abbott RD, Ross GW, Petrovitch H, et al. Bowel movement frequency in late-life and incidental Lewy bodies. Mov Disord 2007;22:1581-1586.

9. Cersosimo MG, Benarroch EE. Pathological correlates of gastrointestinal dysfunction in parkinson's disease. Neurobiol Dis 2012;46:559-564.

10. Böttner M, Zorenkov D, Hellwig I, et al. Expression pattern and localization of alpha-synuclein in the human enteric nervous system. Neurobiol Dis 2012;48:474-480.

11. Gray MT, Munoz DG, Gray DA, Schlossmacher MG, Woulfe JM. Alpha-synuclein in the appendiceal mucosa of neurologically intact subjects. Mov Disord 2014;29:991-998.

12. Visanji NP, Marras C, Kern DS, et al. Colonic mucosal a-synuclein lacks specificity as a biomarker for parkinson disease. Neurology 2015;84:609616.

13. Chung SJ, Kim J, Lee HJ, et al. Alpha-synuclein in gastric and colonic mucosa in parkinson's disease: limited role as a biomarker. Mov Disord 2016;31:241-249.

14. Song KH, Jung HK, Min BH, et al. Development and validation of the Korean Rome III questionnaire for diagnosis of functional gastrointestinal disorders. J Neurogastroenterol Motility 2013;19:509-515.

15. Gibb WR, Lees AJ. The relevance of the lewy body to the pathogenesis of idiopathic parkinson's disease. J Neurol Neurosurg Psychiatry 1988;51:745-752.

16. Rocca WA, Maraganore DM, McDonnell SK, Schaid DJ. Validation of a telephone questionnaire for parkinson's disease. J Clin Epidemiol 1998;51:517-523.

17. Drossman DA. The functional gastrointestinal disorders and the Rome III process. Gastroenterology 2006;130:1377-1390.

18. Chaudhuri KR, Martinez-Martin P, Schapira AH, et al. International multicenter pilot study of the first comprehensive self-completed nonmotor symptoms questionnaire for parkinson's disease: the NMSQuest study. Mov Disord 2006;21:916-923.

19. Ryu HS, Choi SC. Recent updates on the treatment of constipation. Intest Res 2015;13:297-305.

20. Cersosimo MG, Benarroch EE. Neural control of the gastrointestinal tract: implications for parkinson disease. Mov Disord 2008;23:10651075.

21. Furness JB. The enteric nervous system: normal functions and enteric neuropathies. Neurogastroenterol Motil 2008;20(suppl 1):32-38.

22. Lebouvier T, Neunlist M, Bruley des Varannes S, et al. Colonic biopsies to assess the neuropathology of parkinson's disease and its relationship 
with symptoms. PloS One 2010;5:e12728.

23. Beach TG, Adler CH, Sue LI, et al. Multi-organ distribution of phosphorylated alpha-synuclein histopathology in subjects with lewy body disorders. Acta neuropathol 2010;119:689-702.

24. Annerino DM, Arshad S, Taylor GM, Adler CH, Beach TG, Greene JG. Parkinson's disease is not associated with gastrointestinal myenteric ganglion neuron loss. Acta neuropathol 2012;124:665-680.

25. Gold A, Turkalp ZT, Munoz DG. Enteric alpha-synuclein expression is increased in parkinson's disease but not alzheimer's disease. Mov Disord 2013;28:237-240

26. Braak H, Sastre M, Bohl JR, de Vos RA, Del Tredici K. Parkinson's disease: lesions in dorsal horn layer $\mathrm{I}$, involvement of parasympathetic and sympathetic pre- and postganglionic neurons. Acta neuropathol 2007;113:421-429.

27. VanderHorst VG, Samardzic T, Saper CB, et al. alpha-synuclein pathology accumulates in sacral spinal visceral sensory pathways. Ann Neurol 2015;78:142-149.

28. Dickson DW, Fujishiro H, DelleDonne A, et al. Evidence that incidental lewy body disease is pre-symptomatic parkinson's disease. Acta neuropathol 2008;115:437-444.

29. Benarroch EE, Schmeichel AM, Sandroni P, Low PA, Parisi JE. Involvement of vagal autonomic nuclei in multiple system atrophy and lewy body disease. Neurology 2006;66:378-383.

30. Marrinan S, Emmanuel AV, Burn DJ. Delayed gastric emptying in parkinson's disease. Mov Disord 2014;29:23-32.

31. Peelaerts W, Bousset L, Van der Perren A, et al. $\alpha$-Synuclein strains cause distinct synucleinopathies after local and systemic administration. Nature 2015;522:340-344.

32. Stokholm MG, Danielsen EH, Hamilton-Dutoit SJ, Borghammer P. Pathological $\alpha$-synuclein in gastrointestinal tissues from prodromal parkinson disease patients. Ann Neurol 2016;79:940-949.

33. Hilton D, Stephens M, Kirk L, et al. Accumulation of $\alpha$-synuclein in the bowel of patients in the pre-clinical phase of Parkinson's disease. Acta neuropathol 2014;127:235-241.

34. Lebouvier T, Chaumette T, Damier P, et al. Pathological lesions in colonic biopsies during parkinson's disease. Gut 2008;57:1741-1743.

35. Pouclet H, Lebouvier T, Coron E, Des Varannes SB, Neunlist M, Derkinderen P. A comparison between colonic submucosa and mucosa to detect lewy pathology in parkinson's disease. Neurogastroenterol Motil 2012;24:e202-e205.

36. Sanchez-Ferro Á, Rábano A, Catalán MJ, et al. In vivo gastric detection of $\alpha$-synuclein inclusions in parkinson's disease. Mov Disord 2015;30:517-524. 